\title{
Genome comparison provides molecular insights into the phylogeny of the reassigned new genus Lysinibacillus
}

Kai Xu $u^{1,2}$, Zhiming Yuan ${ }^{1}$, Simon Rayner ${ }^{3^{*}}$ and Xiaomin $\mathrm{Hu}^{1^{*}}$

\begin{abstract}
Background: Lysinibacillus sphaericus (formerly named Bacillus sphaericus) is incapable of polysaccharide utilization and some isolates produce active insecticidal proteins against mosquito larvae. Its taxonomic status was changed to the genus Lysinibacillus in 2007 with some other organisms previously regarded as members of Bacillus. However, this classification is mainly based on physiology and phenotype and there is limited genomic information to support it.

Results: In this study, four genomes of L. sphaericus were sequenced and compared with those of 24 representative strains belonging to Lysinibacillus and Bacillus. The results show that Lysinibacillus strains are phylogenetically related based on the genome sequences and composition of core genes. Comparison of gene function indicates the major difference between Lysinibacillus and the two Bacillus species is related to metabolism and cell wall/membrane biogenesis. Although L. sphaericus mosquitocidal isolates are highly conserved, other Lysinibacillus strains display a large heterogeneity. It was observed that mosquitocidal toxin genes in L. sphaericus were in close proximity to genome islands (GIs) and mobile genetic elements (MGEs). Furthermore, different copies and varying genomic location of the Gls containing binA/binB was observed amongst the different isolates. In addition, a plasmid highly similar to pBsph, but lacking the Gl containing binA/binB, was found in L. sphaericus SSII-1.
\end{abstract}

Conclusions: Our results confirm the taxonomy of the new genus Lysinibacillus at the genome level and suggest a new species for mosquito-toxic L. sphaericus. Based on our findings, we hypothesize that (1) Lysinibacillus strains evolved from a common ancestor and the mosquitocidal L. sphaericus toxin genes were acquired by horizontal gene transfer (HGT), and (2) capture and loss of plasmids occurs in the population, which plays an important role in the transmission of binA/binB.

Keyword: Lysinibacillus, Bacillus, Lysinibacillus sphaericus, Genome, Phylogeny

\section{Background}

Lysinibacillus sphaericus (formerly named Bacillus sphaericus) is a Gram-positive, aerobic, mesophilic, and sporeforming bacterium that is commonly isolated from soil. It is also an archaic organism whose spores have even been found in 25-40-million-year-old amber [1]. L. sphaericus has very distinctive phenotypic properties, including an inability to utilize polysaccharide pathways and employment of exclusive metabolic pathways for synthesis of a wide

\footnotetext{
* Correspondence: simon.rayner.cn@gmail.com; huxm@wh.iov.cn

${ }^{3}$ State Key Laboratory of Virology, Wuhan Institute of Virology, Chinese

Academy of Sciences, Wuhan 430071, China

'Key Laboratory of Agricultural and Environmental Microbiology, Wuhan Institute of Virology, Chinese Academy of Sciences, Wuhan 430071, China Full list of author information is available at the end of the article
}

variety of organic compounds and amino acids [2]. Some strains produce active insecticidal proteins against mosquito larvae, and thus have been widely used as biocontrol agents for disease-transmitting mosquitoes [3]. The mosquitocidal properties are associated with the sporulationspecific binary toxin (Bin proteins) and vegetative-specific Mtx toxins [4], as well as a novel two-component toxin (Cry48 and Cry49 proteins) produced during sporulation [5]. Compared with another mosquito pathogen, Bacillus thuringiensis subsp. israelensis, L. sphaericus demonstrates a higher efficiency for killing mosquito larvae and a better persistence in the field [6].

The evolutionary model and systematic classification of L. sphaericus continues to be debated. On the basis of flagellar agglutination, L. sphaericus isolates can be grouped 
into 49 serotypes [7]. According to DNA homology between strains, five major groups (I to V) are indicated, each probably corresponding to a separate species because of the relatively low level of homology between groups [8]. However, relatively few biochemical and morphological tests are available to distinguish $L$. sphaericus as a different species. Recently, a multi-locus sequence typing (MLST) study has indicated that the mosquitocidal strains are highly conserved and appear near-clonal [9]. This is consistent with a previous report which observed that toxic $L$. sphaericus strains are all found within DNA subgroup IIA, although in association with nine serotypes $(\mathrm{H} 1, \mathrm{H} 2, \mathrm{H} 3$, $\mathrm{H} 5, \mathrm{H} 6, \mathrm{H} 9, \mathrm{H} 25$, H26, and H48).

In 2007, Bacillus sphaericus was formally renamed $L$. sphaericus and, together with Lysinibacillus boronitolerans and Lysinibacillus fusiformis (formerly named Bacillus fusiformis), was proposed to belong to a novel genus named Lysinibacillus gen. nov. Since then, more and more novel isolates have been assigned to Lysinibacillus. The species classification was mainly based on common features in physiology and phenotype, e.g. Gram-positive, sporeforming, rod-shaped, motile, presence of the Lys-Asp type of peptidoglycan in the cell wall, the main fatty acids as iso$\mathrm{C}_{15: 0}$, and the predominant menaquinones as MK-7 [10], but there is little evidence to support this classification on a genomic basis. Thus, there is a need to analyze the relationship between Lysinibacillus and Bacillus on the genomic level, and to understand the evolution of mosquitocidal $L$. sphaericus.

Although a broad spectrum of data has been collected for $L$. sphaericus, there is limited genome sequence available. One complete genome sequence is available for mosquitocidal strain C3-41 (accession numbers CP000817 and CP000818) [11], and two gapped genome sequences from reference strains KCTC 3346 (or ATCC14577) and OT4b.31(both non-toxic) have also been published $[12,13]$. In this study we report genome sequences of four L. sphaericus strains, comprising three toxic strains (2297, LP1-G, SSII-1) and one non-toxic strain (NRS1693). We also investigate their phylogenetic relationship with genome sequences for Lysinibacillus and Bacillus strains. Our results provide the first support for the taxonomy of the reassigned new genus Lysinibacillus at the genome level and suggest a new species for mosquitocidal L. sphaericus, providing new insight into the evolution of Lysinibacillus.

\section{Results}

\section{General features}

The whole genomes of L. sphaericus 2297, LP1-G, SSII-1 and NRS1693 were sequenced and assembled into 278, 143, 138 and 546 contigs, respectively. An additional 24 genome sequences were selected for comparison to create a final dataset of 28 genomes; 10 came from Lysinibacillus (seven L. sphaericus, two L. fusiformis and one L. boronitolerans), one from Lysinibacillus-related strain Bacillus sp. NRRL B14905 [11], and 17 from the B. cereus group and B. subtilis. The characteristics of all these genomes are summarized in Table 1.

The total genome sizes vary from 4.0 to $6.7 \mathrm{Mb}$ across species and strains. All Lysinibacillus strains have larger chromosome sizes $(4.5 \sim 4.8 \mathrm{M})$ compared to B. subtilis $(4.0 \sim 4.2 \mathrm{M})$ but smaller sizes compared to $B$. cereus group strains $(5.2 \sim 6.7 \mathrm{M})$. Conversely, their $\mathrm{G}+\mathrm{C}$ content $(\sim 37 \%)$ is higher than that $B$. cereus group strains ( $35 \%)$ but lower than that of B. subtilis ( 43\%).

The numbers of predicted genes in L. sphaericus genomes varied from 4,470 to 4,701 , but is likely a factor of incomplete genome assemblies as well as individual strain differences. With C3-41 as a reference, the predicted gene numbers of other L. sphaericus strains varied from 2,791 to 4,202 , corresponding to 62.8 to $90.4 \%$ of the total gene numbers of the individual genome. The novel strains presented in this study (2297, LP1-G, SSII-1 and NRS1693) harbor over $80 \%$ genes predicted to be homologous to genes in C3-41, whereas the corresponding numbers in the two L. sphaericus reference strains KCTC3346 and OT4b.31 were only $62.8 \%$ and $65.4 \%$ respectively.

\section{Phylogenetic relationship}

The Gegenees software package [14] was used for the comparative analysis of the gene content of the 28 genomes. The software resolves each genome into a series of overlapping fragments and then performs pairwise comparison of each fragmented genome. In this way, a distance matrix based on shared fragments is created. A heatmap of the calculated similarity matrix is shown in Figure 1. A number of genomes are well clustered, in particular the toxic isolates of $L$. sphaericus are highly conserved with $>97 \%$ conservation between 2297, LP1-G, SSII-1 and C3-41 (green square towards the top left of the heat map in Figure 1), and clearly distinct from the nontoxic L. sphaericus isolates NRS1693, KCTC_3346 and OT4b.31 (extreme top right in the heatmap). The marine Bacillus spp. NRRL B-14905 isolate showed 79.5\% similarity with the toxic isolates and $55-62 \%$ similarity with the non-toxic strains. This suggests that this marine strain has a taxonomic status that is somewhere between the toxic and non-toxic strains, but closer to the former. In addition, L. fusiformis and L. boronitolerans are related with a similarity of $84 \%$.

Based on the distance matrix Nexus file exported from Gegenees, a dendrogram was produced using SplitsTree 4 (using the neighbor joining method) (Figure 1 left). The tree classifies all Lysinibacillus genomes into two main clusters. The $L$. sphaericus toxic isolates and the marine Bacillus spp. NRRL B-14905 are clustered and closer to $B$. cereus group strains, whereas the three non-toxic $L$. sphaericus strains are clustered with $L$. fusiformis and $L$. 
Table 1 Strains and genome information used in this study

\begin{tabular}{|c|c|c|c|c|c|c|}
\hline Strain & Status & Genome size (bp) & GC content (\%) & No. of contigs & No. of proteins & Genbank accession No. \\
\hline \multicolumn{7}{|l|}{ B. subtilis } \\
\hline QB928 & complete & $4,146,839$ & 43.60 & - & 4,031 & NC_018520 \\
\hline BAB-1 & complete & $4,021,944$ & 43.89 & - & 4,003 & NC_020832 \\
\hline BSn5 & complete & $4,093,599$ & 43.84 & - & 4,145 & NC_014976 \\
\hline 168 & complete & $4,215,606$ & 43.91 & - & 4,003 & NC_000964 \\
\hline 6051-HGW & complete & $4,215,610$ & 43.51 & - & 4,187 & NC_020507 \\
\hline \multicolumn{7}{|l|}{ B. thuringiensis } \\
\hline BMB171 & complete & $5,330,088$ & 35.17 & - & 5,352 & NC_014171 \\
\hline Al Hakam & complete & $5,257,091$ & 35.43 & - & 4,798 & NC_008600 \\
\hline IBL 200 & draft & $6,731,790$ & 34.53 & 2 & 6,693 & NZ_CM000758 \\
\hline HD-789 & complete & $5,495,278$ & 35.17 & - & 6,462 & NC_018508 \\
\hline Bt407 & complete & $5,500,501$ & 35.02 & - & 6,402 & NC_018877 \\
\hline \multicolumn{7}{|l|}{ B. anthracis } \\
\hline Ames & complete & $5,227,293$ & 35.38 & - & 5,039 & NC_003997 \\
\hline \multicolumn{7}{|l|}{ B. cereus } \\
\hline ATCC 14579 & complete & $5,411,809$ & 35.29 & - & 5,231 & NC_004722 \\
\hline $\mathrm{AH} 187$ & complete & $5,269,030$ & 35.51 & - & 5,783 & NC_011658 \\
\hline E33L & complete & $5,300,915$ & 35.13 & - & 5,641 & NC_006274 \\
\hline 03BB102 & complete & $5,269,628$ & 35.33 & - & 5,606 & NC_012472 \\
\hline $\mathrm{AH} 820$ & complete & $5,302,683$ & 35.31 & - & 5,810 & NC_011773 \\
\hline biovar anthracis str. $\mathrm{Cl}$ & complete & $5,196,054$ & 35.25 & - & 5,558 & NC_014335 \\
\hline \multicolumn{7}{|l|}{ L. sphaericus } \\
\hline C3-41 & complete & $4,639,821$ & 37.13 & - & $4,584(4,584)^{*}$ & NC_010382 \\
\hline 2297 & draft & $4,525,834$ & 37.12 & 278 & $4,539(4,102)^{*}$ & JPDJ00000000 \\
\hline LP1-G & draft & $4,542,839$ & 37.20 & 143 & $4,630(4,086)^{*}$ & JPDL00000000 \\
\hline SSII-1 & draft & $4,651,985$ & 37.01 & 138 & $4,701(4,202)^{*}$ & JPDK00000000 \\
\hline NRS1693 & draft & $4,640,690$ & 37.55 & 546 & $4,645(3,817)^{*}$ & JPDM00000000 \\
\hline KCTC 3346 & draft & $4,560,870$ & 37.10 & 83 & $4,443(2,791)^{*}$ & AUOZO0000000 \\
\hline OT4b.31 & draft & $4,856,302$ & 37.51 & 94 & $4,575(3,074)^{*}$ & AQPX00000000 \\
\hline \multicolumn{7}{|l|}{ L. fusiformis } \\
\hline ZB2 & draft & $4,550,616$ & 37.31 & 59 & 4,494 & AMQZO0000000 \\
\hline ZC1 & draft & $4,649,417$ & 37.30 & 113 & 4,729 & ADJR00000000 \\
\hline L. boronitoleransF1182 & draft & $4,461,358$ & 37.49 & 309 & 5,270 & AJXM00000000 \\
\hline Bacillus sp. NRRL B-14905 & draft & $4,497,271$ & 37.56 & 99 & 4,470 & NZ_AAXV00000000 \\
\hline
\end{tabular}

*Number of predicted genes matched with those of L. sphaericus C3-41 genome.

boronitolerans. Thus the L. sphaericus strains are diverse and scattered at the genomic level.

In addition, the genomes of Solibacillus silvestris [GenBank: NC_018065], Sporosarcina pasteurii [GenBank: AYOX00000000], and Ureibacillus thermosphaericus [GenBank: AJIK00000000], which are thought to be sphaericus-like organisms close to L. sphaericus based on $16 \mathrm{~s}$ rDNA and phenotypic analysis [15] were investigated. The results showed that these sphaericus-like organisms were quite divergent at the genome level and there is no obvious relationship with Lysnibacillus and Bacillus (data not shown).

\section{Core conserved genes consensus tree}

As a second estimate of the evolutionary relationship amongst the selected genomes, 55 core genes identified by BLAST analysis (e-value $\leq 1 \mathrm{e}-10$, identity $\geq 0.75$, coverage $\geq$ 0.75) (See Additional file 1: Table S1) were used to generate a consensus phylogenetic tree using the NJ method (Figure 2). Consistent with the previous results, all the 10 


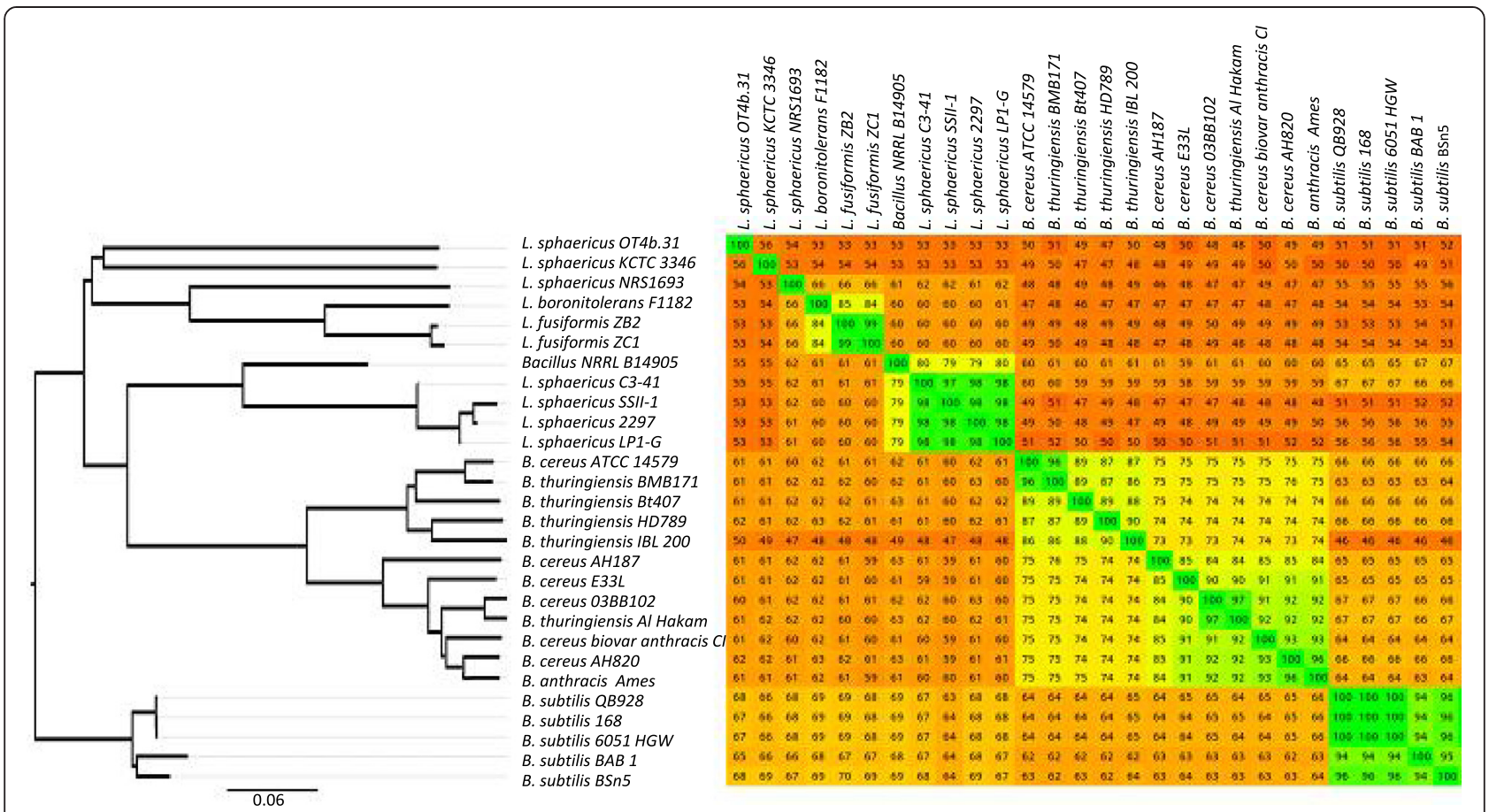

Figure 1 Gegenees analysis of genome composition of 28 genome sequences (10 Lysinibacillus, one from Lysinibacillus-related strain Bacillus sp. NRRL B-14905, and 17 from B. cereus group and B. subtilis, See Table 1 for full details). Right: heat map showing pairwise comparison of each genome pair based on similarity of fragments generated by sliding window. Plot colors reflect the similarity, ranging from low (red) to high (green). The heatmap is asymmetric because the contents of genomes differ in sizes and a similarity is calculated as a fraction of similar sequences in each genome. Left: SplitsTree dendrogram using the Nexus file exported from Gegenees. The toxic L. sphaericus strains form a single well defined tight cluster in both the heatmap and the dendogram (green square towards the top left of heatmap), and are distinct from other strains. The scale bar represents a $6 \%$ difference in average BLASTN score similarity.

Lysinibacillus strains and Bacillus spp. NRRL B-14905 were grouped into one cluster, and the toxic $L$. sphaericus strains and $L$. fusiformis and L. boronitolerans each formed well supported subclusters. However, the non-toxic L. sphaericus strains fail to cluster and are scattered within the Lysinibacillus clade.

\section{Gene content of pan- and core genomes}

To gain further insight into the relationship between the members of Lysinibacillus and Bacillus, the pan- and core genomes, which provides a measure for the intra-species variation in gene content, were each calculated using the PanGP software package $[16,17]$. Since the results above indicate that the B. cereus group and B. subtilis are not closely related, their pan- and core genomes were estimated individually. The resulting plots are shown in Figure 3 and highlight the differences amongst these three groups. The largest difference between the pan- and core genome is seen in Lysinibacillus, with the largest pan-genome (12,365) and the smallest core genome $(2,113)$, indicating the high diversity of the genome set. The $B$. cereus group contains 12 genomes and shows the largest gene number $(4,736$ $6,693)$, but possesses a smaller pan-genome $(11,069)$ and larger core genome $(3,030)$ compared to Lysinibacillus. The
B. subtilis genomes displays the smallest difference between pan $(4,666)$ and core genome $(3,387)$.

In the pan-genomes, the shared genes between Lysinibacillus and the $B$. cereus group $(1,693)$ is greater than the number of genes shared between Lysnibacillus and B. subtilis $(1,307)$ or between $B$. cereus group and B. subtilis $(1,675)$. For the core genome, the shared genes between $B$. cereus group and $B$. subtilis $(1,304)$ is much more than between Lyninibacillus and the other two Bacillus species (815 and 873, respectively) (Figure 4).

\section{Function features of the pan- and core genomes}

To investigate the functional characteristics of the pan and core genomes, the COG (Clusters of Orthologous Groups) database was used to investigate the distribution of pan and core proteins mapping to each COG category for each species group. A plot of protein proportion versus COG function by species/group is shown in Figure 5 . The primary differences are observed in COG categories related to metabolism. For category G (carbohydrate transport and metabolism) the pan- and core genomes sort by protein proportion in the order B. subtilis $>$ B. cereus group $>$ Lysinibacillus. Conversely, for category E (amino acid transport and metabolism) the order is reversed, with 


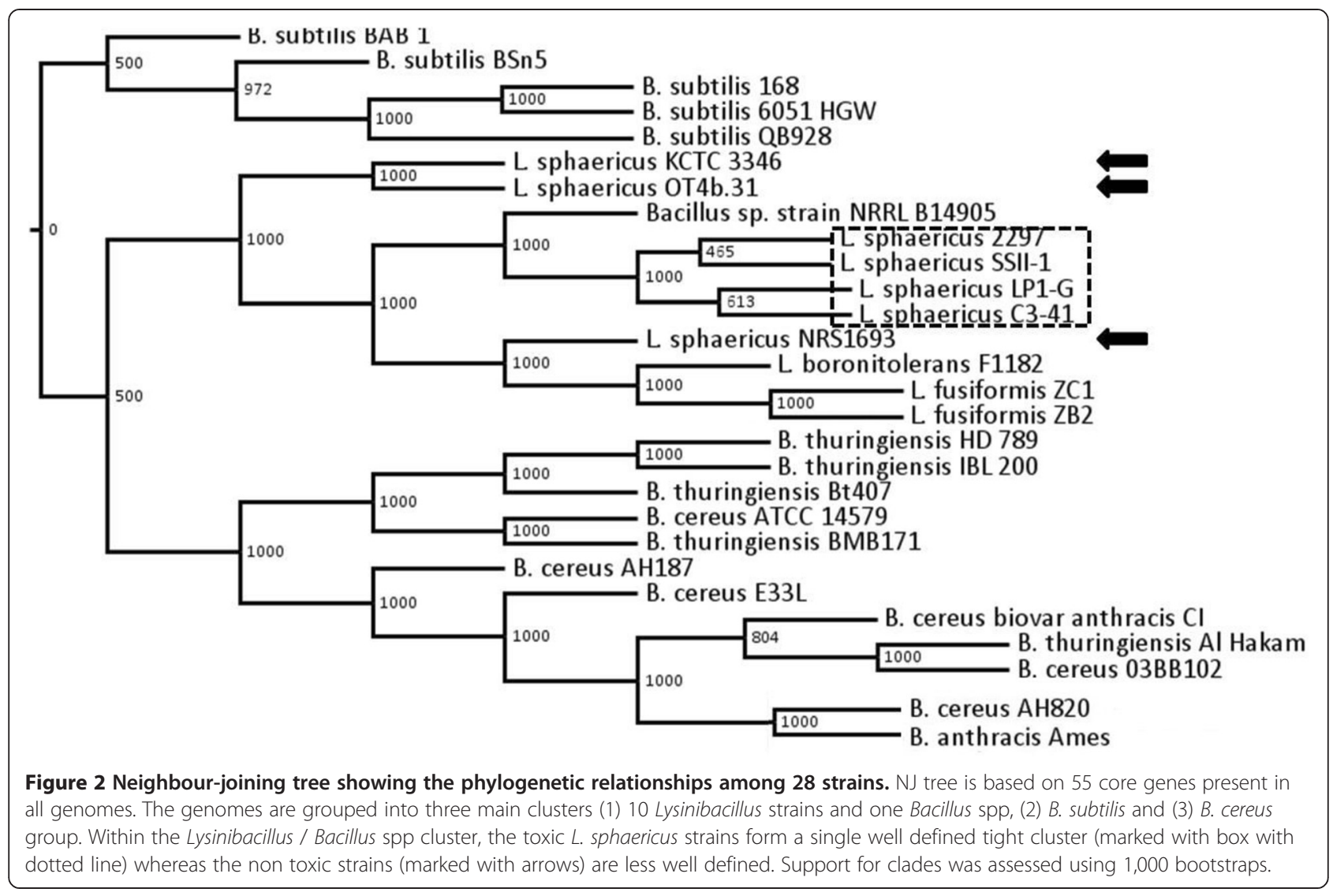

the largest proportion observed in Lysinibacillus, followed by the B. cereus and B. subtilis. For the remaining classifications, the distributions of category $C$ (Energy production and conservation), F (Nucleotide transport and metabolism), $\mathrm{H}$ (Coenzyme transport and metabolism), and Q (Secondary metabolites biosynthesis, transport and catabolism) both the pan- and in the core genomes of Lysinibacillus, were observed to be similar to those of the B. cereus group, but different to B. subtilis. A shift was observed within a genus or species for the core genome compared to the pan genome with a slight overrepresentation of
COG categories related to metabolism, except G, Q (Secondary metabolites biosynthesis, transport and catabolism), and P (Inorganic ion transport and metabolism). This indicates that the gene content for metabolism of amino acids, nucleotides, coenzymes, and lipids is more conserved than for carbohydrates, secondary metabolites and inorganic ions.

Differences in the distribution of the COG categories involved in cellular process and signaling were also observed. For instance, Lysinibacillus displays larger proportions for category T (Signal transduction mechanisms) and N (Cell
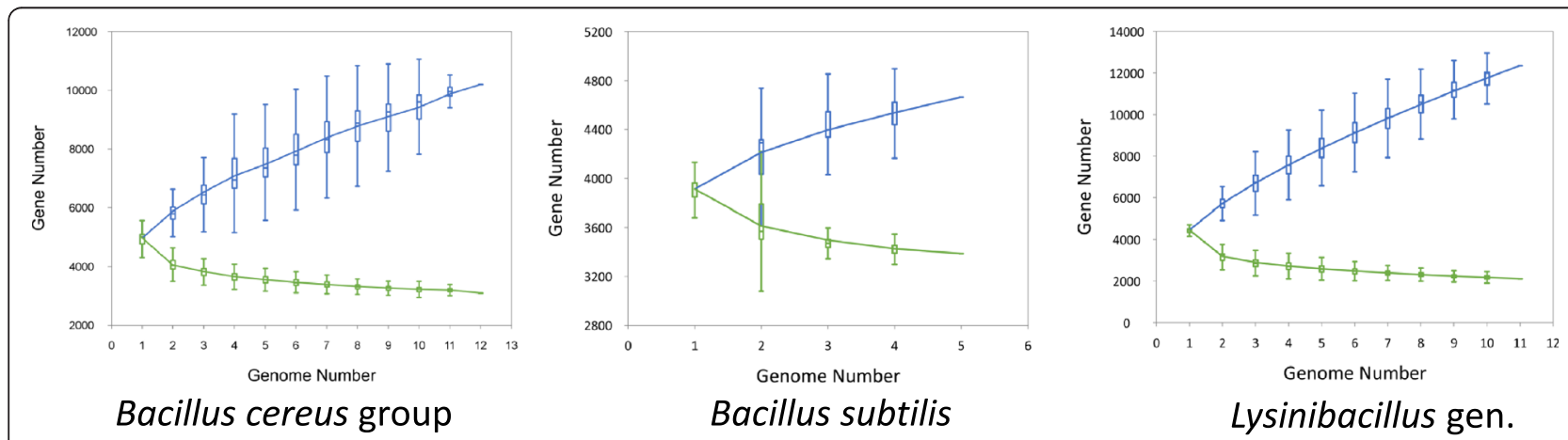

Figure 3 Pan- and core genome plots. (a) B. cereus group, (b) B. subtilis and (c) Lysinibacillus genomes. The blue (upper) and green (lower) curves represent pan- and core genomes respectively. Each pan- or core genome was identified using permutations of strains of each species. 


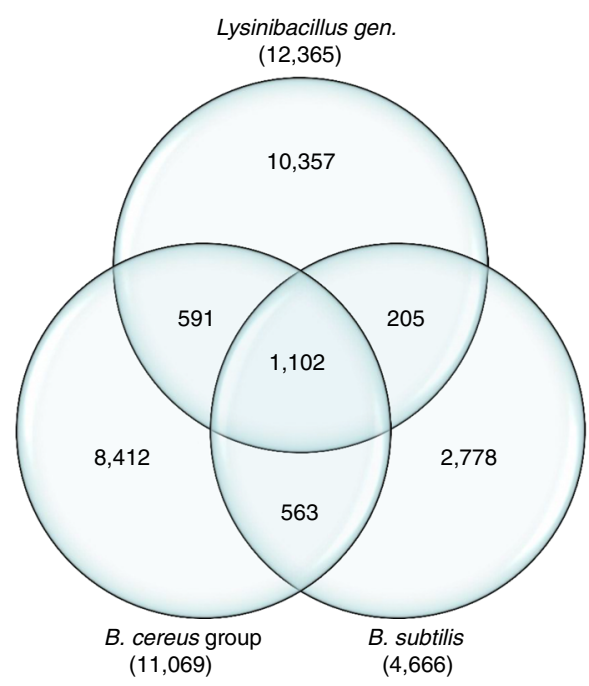

A

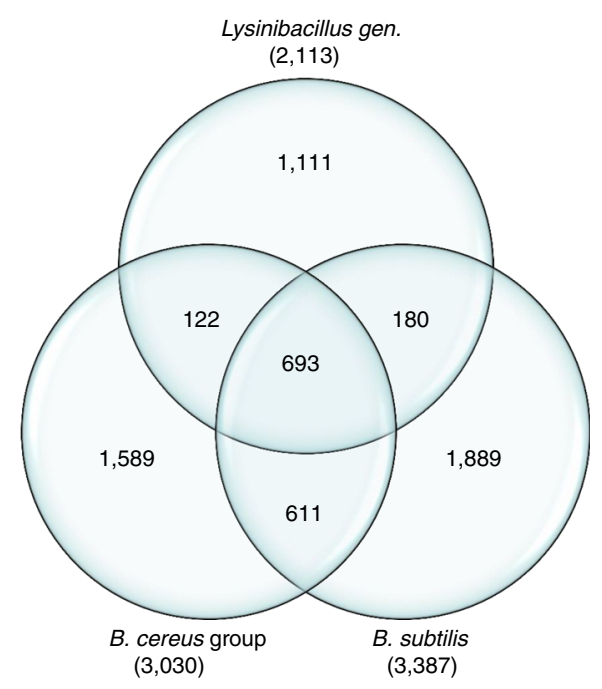

B

Figure 4 Overlap and differences of pan and core genomes amongst the full genome set. Venn diagrams show the overlap and difference between the (A) pan-genome and (B) core genome amongst Lysinibacillus, B. cereus group and B. subtilis.

motility) both in the pan- and in the core genome compared to the B. cereus group and B. subtilis. Also, Lysinibacillus harbors the smallest distribution of category M (Cell wall/ membrane biogenesis) features, which is almost identical in its pan- and core genome, whereas a shift was observed in $B$. cereus group and in B. subtilis, with a slight overrepresentation in the pan genome compared to the core genome.

A subset of COG proteins that were unique in both the pan and core genome of Lysinibacillus were also identified (Additional file 2: Table S2) which is probably related to species-specific characteristics. For instance, six proteins were related to ethanolamine utilization, two proteins were associated with the carbon dioxide concentrating mechanism, six were involved in cobalamin (vitamin B12) biosynthesis, one was related with the cell mobility and one with chromosome segregation.

\section{Characterization of gene contents of Lysinibacillus strains}

Pairwise comparison of the genomes of all the Lysinibacillus strains indicate a strong syntenic relationship with L. sphaericus C3-41 (Additional file 3: Figure S1), indicating that Lysinibacillus strains may have shared a common "chromosome backbone" in a very ancient stage.

The unique genes in the 11 Lysinibacillus strains, varying from 34 to 711 , were COG categorized (data not shown), and appear to reflect observed functional diversity for each strain. For instance, OT4b.31 displayed a large number of unique genes encoding proteins which may be related to its tolerance for heavy-metals, e.g. $\mathrm{Co} / \mathrm{Zn} / \mathrm{Cd} /$ $\mathrm{Mg} / \mathrm{Ni}$ cation transporters (6 genes), metal-dependent hydrolases (3 genes), membrane proteins related to metalloendopeptidases (3 genes), Zinc metalloprotease, $\mathrm{Mn}$-containing catalase, Fe-S cluster formation, and other related Oxidoreductases. KTCC 3346 contained 19 unique genes related to cell wall/membrane/envelope biogenesis, which may be associated with its ability to produce specific surface layer proteins [18-20]. It was also interesting that a gene homologous to the virion core protein of lumpy skin disease virus was identified in the marine strain Bacillus sp. B14905. In addition, genes encoding unique bacteriophage related proteins were identified in $L$. sphaericus 2297 (4), C3-41 (3), SSII-1 (4), OT4b.31 (11), and KCTC 3346 (7), indicating the presence of different bacteriophage(s) or prophage remnants. However, it should be noted that these data are not exact since, with the exception of C3-41, the genomes are not completely sequenced.

A previous study showed that many strains of $L$. sphaericus produce restriction endonucleases which could form a barrier to genetic manipulation [21]. The restriction enzymes and DNA methyltransferases (R-M systems) of the 11 Lysnibacillus strains were predicted by REBASE (http:// rebase.neb.com). The result showed that the R-M systems in the L. sphaericus strains all belong to type II. C3-41 has the most abundant genes encoding DNA methyltransferases, with three on the chromosome and three on the plasmid pBsph, whereas 2297 and OT4b.31 only have one.

\section{Evolution of mosquitocidal L. sphaericus}

10 genomic islands (GIs) were predicted in the chromosome genome of L. sphaericus C3-41 (Table 2, Figure 6), which are mainly located in the most hypervariable regions of the 


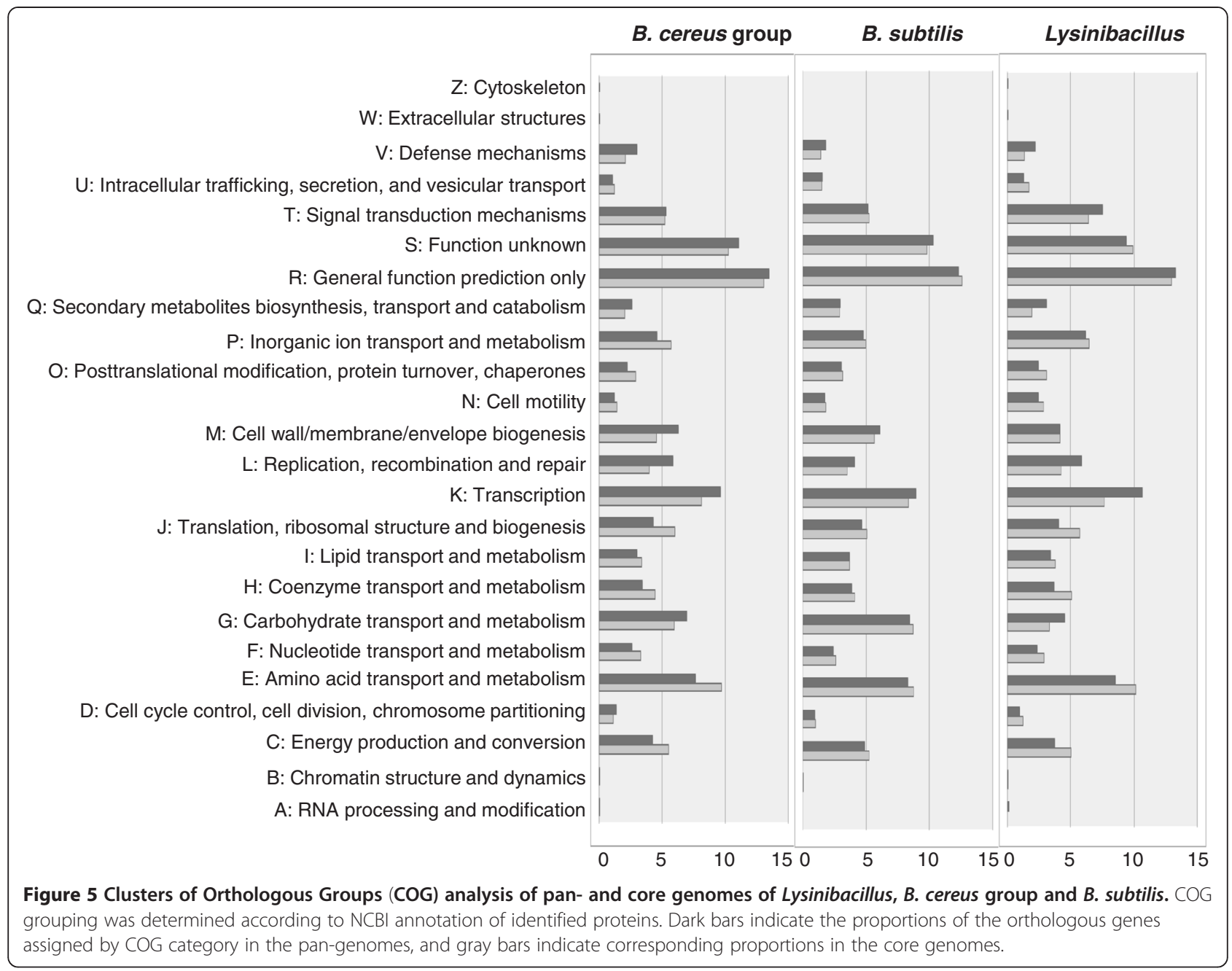

genome, and carry mobile genetic elements (MGEs), such as prophages and transposons, suggesting that these regions are associated with horizontal gene transfer (HGT). It was observed that all the mosquitocidal toxin genes are within (e.g. $m t x 2 / m t x 3$ and $\operatorname{bin} A / \operatorname{bin} B$ ), or close to (e.g. $m t x 1)$ the GIs; furthermore, these toxin genes are flanked by MGEs as previously described [11]. Thus, one possibility is that these mosquitocidal toxin genes were transferred to the common ancestor of $L$. sphaericus through HGT. GI7 (ca. $35 \mathrm{~kb}$ ) consists of binary toxin genes $\operatorname{bin} A$ and $\operatorname{bin} B$, which is the primary genetic basis of the mosquitocidal activity of $L$. sphaericus; this GI was present in C3-41, 2297 and LP1-G. A previous study showed that there are two copies of GI7 in L. sphaericus C3-41, present in both the chromosome and pBsph [11]. However, only one copy of GI7 was found in 2297 and LP1-G. Also, whereas C3-41 has an insert element (named ISBsph9) located downstream of $\operatorname{binA} / \operatorname{binB}$ within GI7, a probable transposase pseudogene is presented in the equivalent region of 2297 and LP1-G.

In addition, a large contig in the genome of strain SSII-1 has a high overlap (>70\%) and similarity (>95\%) with
pBsph, indicating that SSII-1 harbors a pBsph-like plasmid (named pBsph-2). Gene function analysis revealed that this contains some genes involved in replication, recombination and repair, but no GI7 was observed in pBSph-2. It is interesting that the large plasmid pBSph and pBSph-2 contain five genes which are predicted to encode proteins homologous to the type IV secretion system (e.g. VirD4, VirB4, and VirB6) and one gene encoding pilus assembly ATPase, all which may be involved in conjugal transfer. However, the function of the pBsph-2 is still to be characterized.

\section{Discussion}

Lysinibacillus belongs to the family Bacillaceae. Organisms in this genus were previously regarded as members of Bacillus, but their taxonomic status was changed to the genus Lysinibacillus in 2007 [10] and it remains for the classification to be confirmed on a genomic level. Moreover, as an important model bacterium for metabolism and mosquito control, the evolutionary model and systematic classification of $L$. sphaericus is a continual 
Table 2 Genome Islands (GIs) predicted in L. sphaericus C3-41

\begin{tabular}{|c|c|c|c|}
\hline Gls & Containing ORFs & Major Function & Functional categories \\
\hline Gl1 & Bsph1038 Bsph1073 & Cell wall/membrane/envelope biogenesis, Mt×2 & Fitness island \\
\hline Gl 2 & Bsph1085 Bsph1110 & cell division or chromosome partitioning & Fitness island \\
\hline Gl 3 & Bsph $1936 \sim$ Bsph 1953 & Phage remnant & Symbiosis island \\
\hline Gl4 & Bsph2575 Bsph 2615 & Multiple classes, major in information storage and processing & Fitness island \\
\hline Gl5 & Bsph2815 Bsph 2824 & Mosquitocidal toxin & Pathogenicity island \\
\hline Gl6 & Bsph2913 Bsph 2922 & Lipid transport and metabolism & Metabolic island \\
\hline Gl7 & Bsph3179 Bsph 3195 & Mosquitocidal toxin & Pathogenicity island \\
\hline Gl8 & Bsph3265 Bsph 3275 & Poorly characterized* & unknown \\
\hline Gl9 & Bsph3521 Bsph 3538 & Replication, recombination and repair & Fitness island \\
\hline Gl10 & Bsph4022 Bsph 4035 & Poorly characterized* & unknown \\
\hline
\end{tabular}

*Many CDSs have no matches to known function protein.

source of interest and debate. Therefore, exploring the phylogenetic relationship amongst members of the $L$. sphaericus genus in order to confirm the taxonomy of the reassigned new genus Lysinibacillus at the genomic level is of major importance. In this study, several novel genome sequences of $L$. sphaericus are reported, and their phylogenetic relationship with other genome sequences of Lysinibacillus and Bacillus strains are investigated.

The results showed that the genomes of all the studied Lysinibacillus strains and the marine strain Bacillus sp. B14905 show a high syntenic relationship with that of $L$. sphaericus C3-41, indicating these strains may have a common ancestor. Furthermore, the consensus trees based on the core genes and the genomic content indicated all the tested 10 Lysinibacillus organisms and B14905 are phylogenetically related and fall into a distinct and well defined cluster, confirming the taxonomy of the new Lysinibacillus genus. A previous study showed that one subspecies of $B$. subtilis is closely related with $L$. sphaericus based on $16 \mathrm{~s}$ rDNA analysis $[22,23]$. However, at the genome level, Lysinibacillus and $B$. subtilis are clustered separately. Moreover, it is interesting that despite being intergenus of Bacillus, the $B$. cereus group is not closely related to $B$. subtilis.

We also observed that a major difference between $L$. sphaericus and the two Bacillus species is the proportion of proteins encoded by the genome related to metabolism. This is in accordance with the observed speciesspecific metabolic characteristics; Lysinibacillus cannot utilize polysaccharides but alternatively metabolizes a wide variety of organic compounds and amino acids as an energy source [2]. This may explain our observation that, compared to B. cereus group and B. subtilis, Lysinibacillus has an abundance of genes for amino acid transport and metabolism but fewer and less variable genes related to carbohydrate transport and metabolism (probably due to functional degradation). It is interesting to note that all the Lysinibacillus strains have an ethanolamine utilization gene cluster. This could be a complementary pathway for an insect pathogen unable to use polysaccharide for surviving in the insect gut [24]. In addition, a difference was observed in the proportion of proteins with a COG classification of cell wall biosynthesis-related proteins, with members of the $B$. cereus group displaying a larger proportion in the pan genome than that in the core genome. This is probably because some $B$. cereus group strains, e. g. Bacillus mycoids and Bacillus pseudomycoids, have a different cell wall/membrane phenotype [25]. In contrast, the cell wall biosynthesis-related proteins in the pan-genome of Lysinibacillus gen. strains are almost completely complimentary to the set identified in the core genome, suggesting the strains within this genus have specific and common features in their cell wall/membrane composition [10].

Amongst L. sphaericus, the genomes of toxic isolates are highly conserved, whereas those of the non-toxic strains are clearly variant. This confirms a recent MLST study which indicated that there is considerably more heterogeneity amongst non-toxic strains than amongst toxic ones, with the toxic strains tested appearing nearclonal [9]. This is also consistent with a previous study which showed that recombination among L. sphaericus strains was relatively rare compared to the rates for most species, such as the B. cereus group, Campylobacter coli, and Listeria monocytogenes, and suggested that mutations were largely responsible for the generation of sequence diversity in L. sphaericus [9]. Due to the large heterogeneity, it is supposed that the evolutionary distance and timescale of divergence between toxic and non-toxic strains of $L$. sphaericus should be large. In contrast to the lesser variation within a single species in other Bacillus spp., the toxic L. sphaericus strains may be separated from non-toxic strains and we propose a new species should be introduced. 


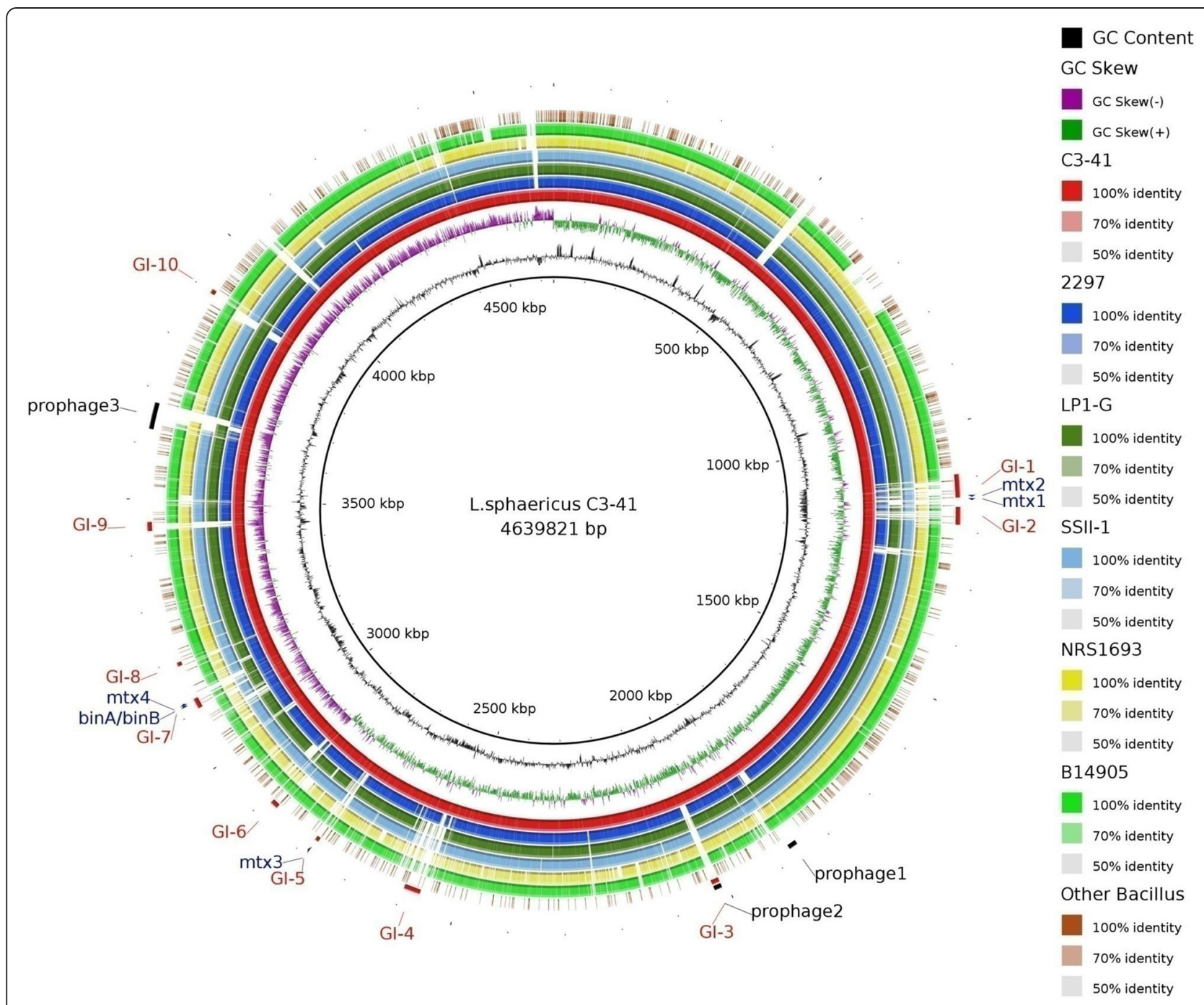

Figure 6 Genome Island (GI) prediction comparative analyses of L. sphaericus genomes. The C3-41 chromosome was used as reference. From the inside: circle 1, genome scale;circles 2 and 3, GC content and GC skew; circles 4-9, genome of C3-41 (red), 2297 (blue) , LP1-G (green), SSII-1 (sky blue), NRS1693 (yellow) and B14905 (emerald green), with colors from dark to light reflecting the similarity from high to low; circle 10, representative genomes for other Bacillus strains (i.e. B. anthracis strain Ames, B. cereus strain AH187, B. thuringiensis strain BMB171, and B. subtilis strain 168) used as outgroups and displaying similar mapping. The predicted Gls, prophages and toxic genes are marked on the outside of the circles.

This raises the question of how L. sphaericus strains obtained mosquitocidal toxin genes and evolved into a separate population. The proximity of mosquitocidal toxin genes with the GIs and the MGEs indicates a HGT origin and the structure of GI7, a pathogenicity island containing the major mosquitocidal toxin gene $\operatorname{bin} A$ / binB and MGEs, provides a possible clue. GI7 possesses multiple genomic locations across the various genomes: it is present in both the chromosome and plasmid of C3-41, but is only found in the chromosome of 2297 and LP1-G, and is absent in SSII-1; furthermore, it is present in pBSph but absent in the highly similar plasmid $\mathrm{pBSph}-2$. In order to assess the basic transfer potential of $\mathrm{pBSph}$ and $\mathrm{pBSph}-2$, homologs of the T4SS genes
virB4, virB6, and virD4 that were identified to be in the transfer region of the conjugative plasmids, e.g. the Ti-plasmid from Agrobacterium tumefaciens, plasmid pIP501 from Enterococcus faecalis, and plasmid pAW63 from $B$. thuringiensis [26,27], were investigated. The result showed that each harbor five T4SS genes displaying low levels of homology to known T4SS genes, making it doubtful that they could function as the concerted secretion machinery required for conjugation. The conjugative and transfer promoting capacities of $\mathrm{pBsph}$ and pBsph-2 were assessed by tri-parental matings as previously described [28]. None were indicative of selfconjugative or mobilizable activities, at least under the conditions used in the assay (detection limit of 10-7 T/ 
R) (data not shown). One interpretation of these results is that the ancestral form of the plasmid was conjugative and genetic drifts in subsequent lineages lead to the loss of transfer capability.

A previous study surveyed the presence of toxin genes and the associated mosquitocidal activities of $L$. sphaericus isolates. It showed that non-toxic strains contain only $m t x 2$ or no toxin gene at all; low toxicity strains possess $m t x 1, m t x 2$ and $m t x 3$; and moderately or highly toxic strains contain $m t x 3$, $\operatorname{bin} A / \operatorname{bin} B$ and/or cry $48 A a /$ cry $49 A a$, in which some isolates also contains $m t x 1$ and $m t x 2$ [9]. In addition, $m t x 2$ and $m t x 3$ are homologous and have close orthologs in Bacillus sp. strain NRRL B14905 [11]. It is also interesting that Mtx2 and Mtx3 are members of Clostridium epsilon toxin ETX/MTX2 family (pfam 03318) of pore forming toxins defined in the NCBI Conserved Domain Database [29]. Combining the results of our analysis with these other findings, we propose the following hypothesis for the evolution of mosquitocidal L. sphaericus: 1) Lysnibacillus strains share a common ancestor; 2) A $m+x 2$ or $m t x 3$ ortholog was initially acquired by HGT; 3 ) The acquisition of $m+x 2 / m t x 3$ was followed by acquisition of $\operatorname{bin} A / \operatorname{bin} B$, cry $48 a /$ cry $49 a$ and $m t x 1$ also by HGT at a later time; 4$)$ The GI containing $\operatorname{bin} A / \operatorname{bin} B$ was obtained by phage integration into the chromosome and/or plasmid; 5) The ancestral form of $\mathrm{pBsph}$ and $\mathrm{pBsph}-2$ was conjugative, whose capture and loss probably occurred in the population, probably playing an important role for the transmission of $\operatorname{bin} A / \operatorname{bin} B$. However, while the data collected to date supports this hypothesis, additional L. sphaericus genomes are needed together with complementary experimental and bioinformatics analysis.

\section{Conclusions}

We present the genome sequences of four Lysinibacillus strains and investigate their phylogenetic relationship to other available Lysinibacillus strains based on analysis of genome structure and identified core genes. Our results provide the first support at the genome level for the classification of these strains into a separate genus. Our analysis also indicates that mosquitocidal $L$. sphaericus isolates appear distinct from other Lysinibacillus organisms at the genome level, suggesting they should be classified into a separate species. Based on our findings, we hypothesis that Lysnibacillus strains evolved from a common ancestor, and the mosquitocidal toxin genes were acquired by horizontal gene transfer (HGT) resulting in the evolution of the mosquitocidal L. sphaericus.

\section{Methods}

\section{Genome sequencing}

Genome sequencing of L. sphaericus 2297, LP1-G, SSII1 and NRS1693 was carried out using an Illumina HiSeq
2000 system by Encode Genomics Bio-Technology Co. (Suzhou, China). Paired-end reads with average length 72 and minimum read quality of 35 were used for assembly using the Velvet-1.0.14 software package [30]. Using the genome sequence of $L$. sphaericus C3-41 [GenBank: CP000817 and CP000818] as reference, strains 2297, LP1-G and SSII-1 showed 91\% coverage, and their assembly produced 278, 143 and 138 contigs respectively. Strain NRS1693 showed $\sim 74 \%$ coverage, and the assembly produced 546 contigs (Table 1 ).

\section{Selection of genomes used in this study}

All the 10 Lysinibacillus genomes available at the time of analysis and one Lysinibacillus-related strain Bacillus sp. NRRL B-14905 [11] were included. The selection of 20 genomes from two representative species of Bacillus, $B$. subtilis and $B$. cereus group, was based on a previous study [23], which showed that B. subtilis is classified into two subspecies and one is closely related with $L$. sphaericus, and that B. cereus group is located on a clade neighboring L. sphaericus/B. subtilis. Thus, the five selected genomes of $B$. subtilis were well representative of the two subspecies. Since the seven members (i.e. B. cereus, $B$. thuringiensis, B. anthracis, B. weihenstephanensis, $B$. mycoides, B. pseudomycoides and B. cytotoxicus) of B. cereus group share close genetic and biochemical relatedness, only 15 genomes of the three major members (i.e. $B$. cereus, B. thuringiensis, B. anthracis) were selected as representative of the strains and species, other closely related or derivative strains were not included. In summary, a total of 17 complete and 11 gapped genomes from Lysinibacillus, B. cereus group, and B. subtilis strains were selected for analysis in this study (Table 1).

In addition, the genomes of Solibacillus silvestris [GenBank: NC_018065], Sporosarcina pasteurii [GenBank: AYOX00000000], and Ureibacillus thermosphaericus [GenBank: AJIK00000000], which are thought to be sphaericus-like organisms close to L. sphaericus based on $16 \mathrm{~s}$ rDNA and phenotypic analysis and previously thought belong to Bacillus [15] were also selected to compare with Lysinibacillus strains.

\section{Genome annotation}

Genome annotation was performed using the xBASE web service (http://www.xbase.ac.uk/annotation/), which comprises the following steps: (i) Glimmer is used for gene prediction; (ii) tRNA genes are predicted using tRNAScan-SE [31]; (iii) ribosomal RNA genes are searched for with RNAmmer [32]; (iv) protein BLAST is run using the translated coding sequences as a query against the reference sequence; (v) the best result for each BLAST search is imported as the gene annotation (if under the user-supplied E-value cutoff) $[33,34]$. Primary parameters were set as default, which sets the minimum length of a gene to be $90 \mathrm{bp}$, 
while the permitted maximum overlap of two genes is $50 \mathrm{bp}$, and the BLAST e-value cutoff is1e-10.

Each annotated protein was then compared to the COG database using BLASTP to identify its member functional groups.

\section{Fragmented alignment of multiple genomes and phylogenomic relationship}

A all-against-all fragment comparison analysis was performed using Gegenees (version 1.1.5) software by fragmenting genomes and comparing all pieces with all genomes [14]. The heat-plot was based on a fragmented alignment using BLASTN with settings 500/500. The cutoff threshold for non-conserved material was $30 \%$. A dendrogram was produced in SplitsTree version 4.12.8 (using the neighbor-joining method) made from a Nexus file exported from Gegenees [35].

Ultra-fast alignments of all Lysinibacillus genomes were finished by the MUMmer program (version 3.0) and the colinearity relationship of each draft genome with C3-41 was calculated [36,37].

\section{Pan- and core genome analysis}

The respective pan- and core genomes of $12 \mathrm{~B}$. cereus group strains, 5 B. subtilis strains and 11 Lysinibacillus strains were calculated using the PanGP software package (http://pangp. big.ac.cn) [16,38], and a BLAST Matrix was constructed using a cutoff of $1 \mathrm{e}^{-10}$, and $50 \%$ identity and coverage. An R-script was used to analyze the COG protein composition in the pan- and core genomes, and the results were visualized in a bar chart [39].

\section{Gene Islands (Gls) prediction}

The GIs in the chromosome of L. sphaericus C3-41 were predicted using IslandViewer (http://www.pathogenomics.sfu.ca/islandviewer/query.php) [40]. Using the C3-41 chromosome as the reference, the draft genome sequences of 2297, LP1-G, SSII-1, NRS1693 and Bacillus sp. NRRL B-14905 were compared and mapped with BRIG (version 0.95) [41] and GBrowse (version 2.49) $[42,43]$, with the complete genomes of $B$. anthracis strain Ames, B. cereus strain AH187, B. thuringiensis strain BMB171, and B. subtilis strain 168 as outgroups. Some distinct special sites, including the predicted GIs, prophages and the mosquitocidal toxin genes were presented graphically outside the circle map.

\section{Nucleotide sequence accession numbers}

All four draft $L$. sphaericus genomes have been deposited at GenBank. Accession numbers are listed in Table 1.

\section{Availability of supporting data}

The data sets supporting the results of this article are included within the article and the additional files.

\section{Additional files}

Additional file 1: Table S1. 55 core genes conserved in Lysinibacillus and Bacillus used to construct the consensus phylogenetic tree in Figure 2.

Additional file 2: Table S2. Predicted COG proteins that are both unique in the pan- and in the core genome of Lysinibacillus.

Additional file 3: Figure S1. MUMmer analysis of the linear relationship between the genome sequence of Lysinibacillus strains and reference strain C3-41. The X-axis represents reference C3-41, and the Y-axis represents each strain of Lysinibacillus. The color bar on the right side reflects the similarity, ranging from high (red) to low (green). All contigs of draft genomes were sorted automatically with C3-41 by MUMmer.

\section{Competing interests}

The authors declare that they have no competing interests.

\section{Authors' contributions}

Conceived and designed the experiments: RS, HX. Performed the experiments: XK. Analyzed the data: XK, RS, HX. Contributed reagents/materials/analysis tools: $X K, R S, H X$. Wrote the paper: XK, YZ, RS, HX. All authors read and approved the final manuscript.

\section{Acknowledgements}

This project was supported by NFSC grants (30800002 and 31272384) and a 973 grant (2009CB118902), China.

\section{Author details}

${ }^{1}$ Key Laboratory of Agricultural and Environmental Microbiology, Wuhan Institute of Virology, Chinese Academy of Sciences, Wuhan 430071, China. ${ }^{2}$ University of the Chinese Academy of Sciences, Beijing 100039, China. ${ }^{3}$ State Key Laboratory of Virology, Wuhan Institute of Virology, Chinese Academy of Sciences, Wuhan 430071, China.

Received: 16 October 2014 Accepted: 19 February 2015

Published online: 27 February 2015

\section{References}

1. Cano RJ, Borucki MK. Revival and identification of bacterial spores in 25- to 40-million-year-old Dominican amber. Science. 1995;268(5213):1060-4.

2. Han B, Liu HZ, Hu XM, Cai YJ, Zheng DS, Yuan ZM. Molecular characterization of a glucokinase with broad hexose specificity from Bacillus sphaericus strain C3-41. Appl Environ Microbiol. 2007;73(11):3581-6.

3. Berry C. The bacterium, Lysinibacillus sphaericus, as an insect pathogen. J Invertebr Pathol. 2012;109(1):1-10.

4. Wirth MC, Yang Y, Walton WE, Federici BA, Berry C. Mtx toxins synergize Bacillus sphaericus and Cry11Aa against susceptible and insecticide-resistant Culex quinquefasciatus larvae. Appl Environ Microbiol. 2007;73(19):6066-71.

5. Jones GW, Nielsen-Leroux C, Yang Y, Yuan Z, Dumas VF, Monnerat RG, et al. A new Cry toxin with a unique two-component dependency from Bacillus sphaericus. FASEB J. 2007:21(14):4112-20.

6. Zhang Y, Liu E, Cai C, Chen Z. Isolation of two highly toxic Bacillus sphaericus strains. Insecticidal Microorg. 1987;1:98-9.

7. Debarjac H, Largetthiery I, Dumanoir VC, Ripouteau H. Serological classification of Bacillus sphaericusstrains on the basis of toxicity to mosquito larvae. Appl Environ Microbiol. 1985;21(1-2):85-90.

8. Krych VK, Johnson JL, Yousten AA. Deoxyribonucleic-acid homologies among strains of Bacillus sphaericus. Int J Syst Bacteriol. 1980;30(2):476-84.

9. Ge Y, Hu XM, Zheng DS, Wu YM, Yuan ZM. Allelic diversity and population structure of Bacillus sphaericus as revealed by multilocus sequence typing. Appl Environ Microbiol. 2011;77(15):5553-6.

10. Ahmed I, Yokota A, Yamazoe A, Fujiwara T. Proposal of Lysinibacillus boronitolerans gen. nov sp nov., and transfer of Bacillus fusiformis to Lysinibacillus fusiformis comb. nov and Bacillus sphaericus to Lysinibacillus sphaericus comb. nov. Int J Syst Evol Microbiol. 2007;57:1117-25.

11. Hu X, Fan W, Han B, Liu H, Zheng D, Li Q, et al. Complete genome sequence of the mosquitocidal bacterium Bacillus sphaericus C3-41 and comparison with those of closely related Bacillus species. J Bacteriol. 2008;190(8):2892-902. 
12. Jeong $H$, Jeong DE, Sim YM, Park SH, Choi SK. Genome sequence of Lysinibacillus sphaericus strain KCTC 3346T. Genome Announc. 2013;1(4):e00625-13.

13. Pena-Montenegro TD, Dussan J. Genome sequence and description of the heavy metal tolerant bacterium Lysinibacillus sphaericus strain OT4b.31. Stand Genomic Sci. 2013;9(1):42-56.

14. Agren J, Sundstrom A, Hafstrom T, Segerman B. Gegenees: fragmented alignment of multiple genomes for determining phylogenomic distances and genetic signatures unique for specified target groups. PLoS One. 2012;7(6):e39107.

15. Nakamura LK. Phylogeny of Bacillus sphaericus-like organisms. Int J Syst Evol Microbiol. 2000;50:1715-22

16. Tettelin H, Riley D, Cattuto C, Medini D. Comparative genomics: the bacterial pan-genome. Curr Opin Microbiol. 2008;11(5):472-7.

17. Zhao $Y$, Jia $X$, Yang J, Ling $Y$, Zhang Z, Yu J, et al. PanGP: A tool for quickly analyzing bacterial pan-genome profile. Bioinformatics. 2014;30(9):1297-9.

18. Cui YB, Zhou Y, Liu WN, Chen QW, Ma GF, Shi WH, et al. Cloning of the surface layer gene sllB from Bacillus sphaericus ATCC 14577 and its heterologous expression and purification. Int J Mol Med. 2012;29(4):677-82.

19. Li J, Yang LL, Hu XM, Zheng DS, Yan JP, Yuan ZM. Nanoscale mono- and multi-layer cylinder structures formed by recombinant S-layer proteins of mosquitocidal Bacillus sphaericus C3-41. Appl Microbiol Biotechnol. 2013;97(16):7275-83.

20. Hu XM, Li J, Hansen BM, Yuan ZM. Phylogenetic analysis and heterologous expression of surface layer protein SlpC of Bacillus sphaericus C3-41. Biosci Biotechnol Biochem. 2008;72(5):1257-63.

21. Zahner V, Priest FG. Distribution of restriction endonucleases among some entomopathogenic strains of Bacillus sphaericus. Lett Appl Microbiol. 1997;24(6):483-7.

22. Xu D, Côté JC. Phylogenetic relationships between Bacillus species and related genera inferred from comparison of $3^{\prime}$ end 165 rDNA and $5^{\prime}$ end 16S-23S ITS nucleotide sequences. Int J Syst Evol Microbiol. 2003;53(3):695-704

23. Porwal S, Lal S, Cheema S, Kalia VC. Phylogeny in aid of the present and novel microbial lineages: diversity in Bacillus. PLoS One. 2009;4(2):e4438.

24. Fox KA, Ramesh A, Stearns JE, Bourgogne A, Reyes-Jara A, Winkler WC, et al. Multiple posttranscriptional regulatory mechanisms partner to control ethanolamine utilization in Enterococcus faecalis. Proc Natl Acad Sci U S A. 2009:106(11):4435-40.

25. Nakamura LK. Bacillus pseudomycoides sp. nov. Int J Syst Bacteriol. 1998:48:1031-5.

26. Abajy MY, Kopec J, Schiwon K, Burzynski M, Doring M, Bohn C, et al. A type IV-secretion-like system is required for conjugative DNA transport of broadhost-range plasmid pIP501 in gram-positive bacteria. J Bacteriol. 2007;189(6):2487-96

27. Lang J, Planamente S, Mondy S, Dessaux Y, Morera S, Faure D. Concerted transfer of the virulence Ti plasmid and companion At plasmid in the Agrobacterium tumefaciens-induced plant tumour. Mol Microbiol. 2013;90(6):1178-89.

28. Hu XM, Van der Auwera G, Timmery S, Zhu L, Mahillon J. Distribution, diversity, and potential mobility of extrachromosomal elements related to the Bacillus anthracis pXO1 and pXO2 virulence plasmids. Appl Environ Microbiol. 2009;75(10):3016-28.

29. Marchler-Bauer A, Lu SN, Anderson JB, Chitsaz F, Derbyshire MK, DeWeeseScott C, et al. CDD: a conserved domain database for the functional annotation of proteins. Nucleic Acids Res. 2011:39:D225-9.

30. Zerbino DR, Birney E. Velvet: algorithms for de novo short read assembly using de Bruijn graphs. Genome Res. 2008;18(5):821-9.

31. Schattner P, Brooks AN, Lowe TM. The tRNAscan-SE, snoscan and snoGPS web servers for the detection of tRNAs and snoRNAs. Nucleic Acids Res. 2005;33(Web Server issue):W686-9.

32. Lagesen $\mathrm{K}$, Hallin $\mathrm{P}$, Rodland EA, Staerfeldt HH, Rognes T, Ussery DW. RNAmmer: consistent and rapid annotation of ribosomal RNA genes. Nucleic Acids Res. 2007;35(9):3100-8.

33. Chaudhuri RR, Pallen MJ. xBASE, a collection of online databases for bacterial comparative genomics. Nucleic Acids Res. 2006;34:D335-7.

34. Chaudhuri RR, Loman NJ, Snyder LAS, Bailey CM, Stekel DJ, Pallen MJ. XBASE2: a comprehensive resource for comparative bacterial genomics. Nucleic Acids Res. 2008:36:D543-6.

35. Huson DH. SplitsTree: analyzing and visualizing evolutionary data. Bioinformatics. 1998;14(1):68-73.
36. Delcher AL, Salzberg SL, Phillippy AM. Using MUMmer to identify similar regions in large sequence sets. Curr Protoc Bioinformatics. 2003; Chapter 10:Unit 10.13.

37. Kurtz S, Phillippy A, Delcher AL, Smoot M, Shumway M, Antonescu C, et al. Versatile and open software for comparing large genomes. Genome Biol. 2004;5(2):R12.

38. Friis C, Wassenaar TM, Javed MA, Snipen L, Lagesen K, Hallin PF, et al. Genomic characterization of Campylobacter jejuni strain M1. PLoS One. 2010;5(8):e12253.

39. Mavromatis K, Ivanova NN, Chen IMA, Szeto E, Markowitz VM, Kyrpides NC The DOE-JGI standard operating procedure for the annotations of microbial genomes. Stand Genomic Sci. 2009;1(1):63-7.

40. Langille MGI, Brinkman FSL. IslandViewer: an integrated interface for computational identification and visualization of genomic islands. Bioinformatics. 2009;25(5):664-5.

41. Alikhan NF, Petty NK, Ben Zakour NL, Beatson SA. BLAST Ring Image Generator (BRIG): simple prokaryote genome comparisons. BMC Genomics, 2011;12:402.

42. Podicheti R, Gollapudi R, Dong Q. WebGBrowse-a web server for GBrowse. Bioinformatics. 2009:25(12):1550-1.

43. Podicheti R, Dong Q. Using WebGBrowse to visualize genome annotation on GBrowse. Cold Spring Harb Protoc. 2010;2010(3):pdb prot5392.

\section{Submit your next manuscript to BioMed Central and take full advantage of:}

- Convenient online submission

- Thorough peer review

- No space constraints or color figure charges

- Immediate publication on acceptance

- Inclusion in PubMed, CAS, Scopus and Google Scholar

- Research which is freely available for redistribution 